\title{
EXPLORING THE DENSITY-DEPENDENT STRUCTURE OF BLOWFLY POPULATIONS BY NONPARAMETRIC ADDITIVE MODELING
}

\author{
Ole C. Linguerde, ${ }^{1}$ Nils Chr. Stenseth, ${ }^{1,4}$ Anja B. Kristoffersen, ${ }^{1}$ Robert H. Smith, ${ }^{2}$ S. Jannicke \\ Moe, ${ }^{1}$ Jonathan M. Read, ${ }^{2}$ Susan Daniels, ${ }^{3}$ And Ken Simkiss ${ }^{3}$ \\ ${ }^{1}$ Department of Biology, University of Oslo, P.O. Box 1050, N-0316 Oslo, Norway \\ 2 Department of Biology, University of Leicester, University Road, Leicester LE1 7RH UK \\ ${ }^{3}$ School of Animal Microbial Sciences, University of Reading, Reading RG6 2AJ UK
}

\begin{abstract}
Abundances of 12 laboratory populations of the greenbottle blowfly (Lucilia sericata) were recorded every two days for $776 \mathrm{~d}$, with separate counts for larvae, pupae, and adults. Half of the populations were exposed to sublethal dosages of the toxic compound cadmium acetate; the remaining populations were considered controls. Initial density was low for half of the populations in each group, and high for the other half. In all populations, the adult abundance underwent sustained fluctuations. However, cadmium-exposed populations had smaller mean larval and adult densities, and fluctuations in adult abundance were less regular than for controls. Data from the first and the second half of the experimental period were analyzed separately in order to assess the effects of possible long-term changes in the dynamics on the estimates. Nonparametric (generalized) additive modeling (GAM) was used to investigate time series dynamics and, in particular, to explore the densityindependent components and the structure of the density-dependent components of the system. Overall, cadmium populations had larger larva-to-adult survival rate and smaller adult survival rate than control populations, and for the second half of the experimental period the reproductive rate was smaller for cadmium populations than for control populations. Estimation of the density-dependent components suggested that survival from larva to adult depended nonlinearly on larval density and that increased larval density had a positive effect on larval survival at low densities. Furthermore, cadmium generally decreased vital rates. However, the analysis suggested that most of the observed differences in dynamical behavior between control and cadmium populations are related to differences in the density-independent components of the demographic rates, rather than differences in the density-dependent structure.
\end{abstract}

Key words: additive models; greenbottle blowfly; cadmium; density-dependent and density-independent components; ecotoxicology; generalized additive model (GAM); Lucilia sericata; nonlinearities; nonparametric regression; population model; time series analysis.

\section{INTRODUCTION}

Nicholson's (1954a, 1957) work on the population dynamics of the sheep blowfly Lucilia cuprina has held a major position in the development of the field of population ecology (Gurney et al. 1980, Begon et al. 1996). Nicholson emphasized the role of populationintrinsic density-dependent factors in determining population dynamic behavior, and his classic laboratory experiments, reported in Nicholson $(1954 a, b)$, were designed to demonstrate what he regarded as a "selfevident truth" (J. L. Readshaw, personal communication). His view has often been contrasted with that of Andrewartha and Birch (1954), who focused on the role of population-extrinsic (generally abiotic) densityindependent factors in determining population dynamic behavior. Today there is general agreement that both density-dependent and density-independent factors are important for understanding the overall dynamics of a

Manuscript received 24 June 1999; revised 27 March 2000; accepted 10 April 2000; final version received 3 October 2000.

${ }^{4}$ Corresponding author. E-mail: n.c.stenseth@bio.uio.no population (Turchin 1995, Leirs et al. 1997). Earlier interpretations of population data largely equated intrinsic population control with stability, and extrinsic, abiotic control with instability and large fluctuations. However, it should never be forgotten that some sort of density dependence is needed for maintaining any population (Orzack 1997). Focusing on the densitydependent structure, May (1976) demonstrated in his pioneering paper that nonlinear density dependencies may give rise to very complicated dynamical behavior in rather simple population models.

Extensive studies of time series data from Nicholson's experiments have generated much valuable insight (e.g., May 1976, Oster 1977, 1981, Gurney et al. 1980, 1983, 1999, Readshaw and Cuff 1980, Readshaw and van Gerwen 1983). In particular, biologically plausible mechanisms capable of explaining the observed cycles in abundances have been described. Much focus has been on revealing dynamic properties of the proposed models (including the location of fixed points and their stability properties), and on relating these properties to biological processes. 
Daniels (1994) reported on a replicated $2 \times 2$ factorial experiment with blowfly populations (Nicholson's $[1954 a, b]$ experiments were not replicated), with larval competition as the main density-dependent factor. Experimental factors were exposure to the toxic heavy metal cadmium (no exposure or a sublethal dose) and initial population density (low or high). Our main interest is in determining whether exposure to cadmium affects vital rates; the initial density factor may be considered a "blocking" variable introduced to increase the sensitivity of the inference for cadmium treatment. Cadmium, an abiotic density-independent factor, is one of the most toxic nonessential elements found in the environment. The main toxic effect is its ability to displace copper and zinc ions from their binding sites in metalloproteins, which destroys the biological properties of many enzymes. Cadmium may also substitute calcium in several physiological processes. Earlier analyses and modeling approaches using these data have been reported by Smith et al. (2000). Here, we report further on the empirical modeling of these population data.

Various statistical approaches have been used to analyze (relatively short) ecological time series, including computer-intensive methods such as nonlinear forecasting and response surface methodology (Sugihara and May 1990, Ellner and Turchin 1995, K.-S. Chan, H. Tong, and N. C. Stenseth, unpublished manuscript). Statistical modeling approaches may broadly be divided into parametric and nonparametric methods (Bowman and Azzalini 1997); the choice between these depends on the purpose of the analysis, as well as on the amount of data available. For answering specific questions about well-defined ecological quantities, parametric models have the benefits of easy interpretation and a well-developed inferential theory. At the first stage of an analysis there may, however, be more emphasis on establishing general features of the dynamical system, like the presence and shape of nonlinearities in the density-dependent structure. In this part of the study, nonparametric models are potentially very useful. Making minimal assumptions about the functional forms, such models allow the data to "speak for themselves". Furthermore, nonparametric methods can be used to test the adequacy of parametric models (Hart 1997). In general, nonparametric estimators may be expected to have small bias and usually a lower order of convergence than their parametric counterparts, hence for small data sets a well-chosen parametric estimator will usually outperform nonparametric estimators in terms of mean square error (Hart 1997). However, it is often difficult to know what an appropriate parametric model would be for a given problem, and nonparametric models may therefore be useful even in situations involving small amounts of data. Here we use a particular class of nonparametric models known as generalized additive models (GAM; e.g., Hastie and Tibshirani 1990; for ecological applications of such

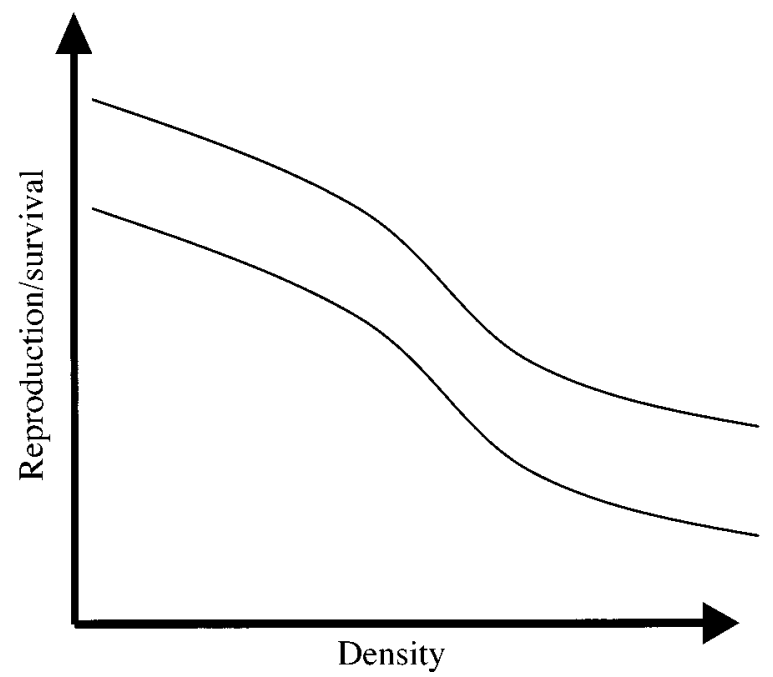

FIG. 1. Demographic rates with identical density-dependent components (nonlinear in this case) and different density-independent components.

models, see, e.g., Stenseth et al. [1997], Bjørnstad et al. [1998]).

As an interpretive aid, it is commonly useful to consider population dynamical behavior as an interaction between a deterministic and a stochastic component (Stenseth et al. 1998). The relative importance of these components is closely related to the issue of densitydependent vs. density-independent control. Typically, within the field of statistical modeling of ecological time series data (Stenseth et al. 1997, Bjørnstad et al. 1998), the skeleton (sensu Tong 1990) represents the deterministic component. The stochastic component is often further divided into (cf. May 1973) demographic stochasticity (typically related to the density-dependent components of the model) and environmental stochasticity (typically related to the density-independent components of the model). In general, the observed population dynamical behavior may be the result of a complex interaction between the deterministic and the stochastic components (Takens 1994, Tong 1995, Wiesenfeld and Moss 1995). For example, in a nonlinear deterministic system generating limit cycles, introducing stochasticity may alter the dominant period of the attractor (Stenseth et al. 1998). As a consequence, one should distinguish between studies aimed at understanding general structural features of the system and the more ambitious task of building a realistic model capturing all observed population dynamic features. Here we restrict ourselves to the former.

Specifically, we aim to elucidate the main characteristics of the demographic rates in the transition between the different life stages. A demographic rate may be decomposed into a density-independent component (a constant for each combination of levels of the density-independent factors) and a density-dependent component (a function of density); Fig. 1 illustrates the 
difference between these two components. The functional shape of the latter component (e.g., whether it is nonlinear) will be a main target in the analysis we present. A secondary aim is to discover whether exposure to a density-independent factor (cadmium) has any effect on the demographic rates. Since cadmium may affect development and survival, increased larval mortality may be experienced at high larval densities, introducing an interacting effect between a densityindependent factor and a density-dependent factor.

\section{The DATA}

Our data represent observations on the temporal fluctuations in 12 laboratory populations of the greenbottle blowfly Lucilia sericata Meigen, the northern hemisphere sister species of L. cuprina, over a period of $776 \mathrm{~d}$. These experiments, carried out at the University of Reading during 1989-1992, are described in detail by Daniels (1994) and Smith et al. (2000). Colonies were kept in separate plastic tanks at $25^{\circ} \mathrm{C}$ and $60 \%$ relative humidity on a $12 \mathrm{~h} / 12 \mathrm{~h}$ light/dark regime. The diet, provided both in fresh and dried form, consisted of $20 \mathrm{~g} / \mathrm{kg}$ agar containing 20\% (by volume) horse blood and $50 \mathrm{~g} / \mathrm{kg}$ dried brewers yeast (20 g fresh diet per population, fully replaced every two days). The fresh diet served both as a medium for oviposition and as food, primarily for the larvae and to some extent also for the adults. Larvae were allowed to migrate into vermiculite surrounding the diet pot for pupating. Sucrose, water and dried diet were provided ad libitum for adults.

The blowfly populations were divided into four experimental groups, each consisting of three replicates. Cadmium acetate was given through fresh and dried diet $(50 \mathrm{mg}$ cadmium $/ \mathrm{kg}$ fresh diet) to six treatment populations; the remaining six populations were considered controls. The three populations in each lowdensity group were initiated with 30 pupae and 30 adults, whereas the three populations in each high-density group were initiated with 150 pupae and 150 adults. In the following, we refer to the populations by the terms control (CON), cadmium (CAD), each subdivided into low density (L) and high density $(\mathrm{H})$ : for example, $\mathrm{CON}(\mathrm{L})$ refers to all three low-density control populations, whereas $\mathrm{CAD}(\mathrm{H} 3)$ refers to the third highdensity cadmium treatment population.

The data consist of a panel with $3 \times 12$ time series, giving counts every two days for three different life stages in each population: larva, pupa, and adult (eggs were not counted). All time series span the same period and were collected by the same personnel. Counts of premigratory larvae and adults include all age groups, whereas pupal counts only include those pupating during the last two days (i.e., since the last count). Only viable pupae (i.e., pupae surviving long enough to become adults) are included in the counts used here (Smith et al. [2000] included both viable and nonviable pupae in their analyses).
Time series data for larvae, pupae, and adults are shown in Figs. 2-4. A qualitative change is seen in some features when comparing control populations (the first two columns of Figs. 2-4) with cadmium populations (the last two columns of Figs. 2-4). Notice that one cadmium population (CAD[L2]) went extinct before the end of the experiment; this population is excluded from our analyses.

In the analyses, combining data from all replicates proved useful, since it led to substantial reduction in the variance of the estimators. However, to evaluate the degree of consistency among the replicates, they were also analyzed separately.

\section{Modeling Stage-Structured Interactions}

Stochastic state transition models (Ebenman and Pearsson 1988) are frequently used to describe temporal population fluctuations (Caswell 1989a, b). Assume for this purpose that the life cycle of the species under consideration is divided into $d$ age classes of equal duration; let $N_{t, 1}, \ldots, N_{t, d}$ denote the population abundances for each age class at time $t$.

The model describing the population-dynamic process relates the state of the system at time $t+1$, defined as $\mathbf{N}_{t+1}=\left(N_{t+1,1}, \ldots, N_{t+1, d}\right)^{\mathrm{T}}$, to earlier states. Analogous to a Leslie matrix representation, this may be expressed as

$$
\mathbf{N}_{t+1}=A\left(\mathbf{N}_{t}, \mathbf{N}_{t-1}, \ldots, \mathbf{N}_{t-q} ; v_{t}\right) \mathbf{N}_{t} .
$$

The transition matrix $A\left(\mathbf{N}_{t}, \mathbf{N}_{t-1}, \ldots, \mathbf{N}_{t-q} ; v_{t}\right)$ may depend on the state of the system at previous time steps $(t, t-1, \ldots, t-q)$ as well as on a stochastic term $v_{t}$. For a given population system, the appropriate form of Eq. 1 will depend on the chosen time unit. Based on the approximation that there are four life stages (larva, pupa, immature adult, and mature adult) each lasting eight days, Smith et al. (2000) use a time step of eight days in their statistical modeling of the same data. Considering the differences in the actual time span of each life stage (see Table 1), a model based on a smaller time step than eight days may be able to capture the dynamics of the system more accurately. Here, we use a time step of two days (i.e., equal to the frequency of observation).

One immediate advantage of using a two-day time step relates to the particular form of our data. In the modeling, we need to take into account the fact that pupal counts represent numbers of individuals that pupate in two-day cohorts, whereas larval and adult counts represent all individuals in a life stage (and thus involve several two-day cohorts). One approach would be to recover the approximate total number of pupae at a given time by summation of several pupal cohort numbers, thus bringing the pupal counts onto the same time scale as the other counts. Modeling and subsequent estimation can then be based exclusively on the total counts of larvae, pupae, and adults. Another approach is to incorporate cohort counts explicitly into 


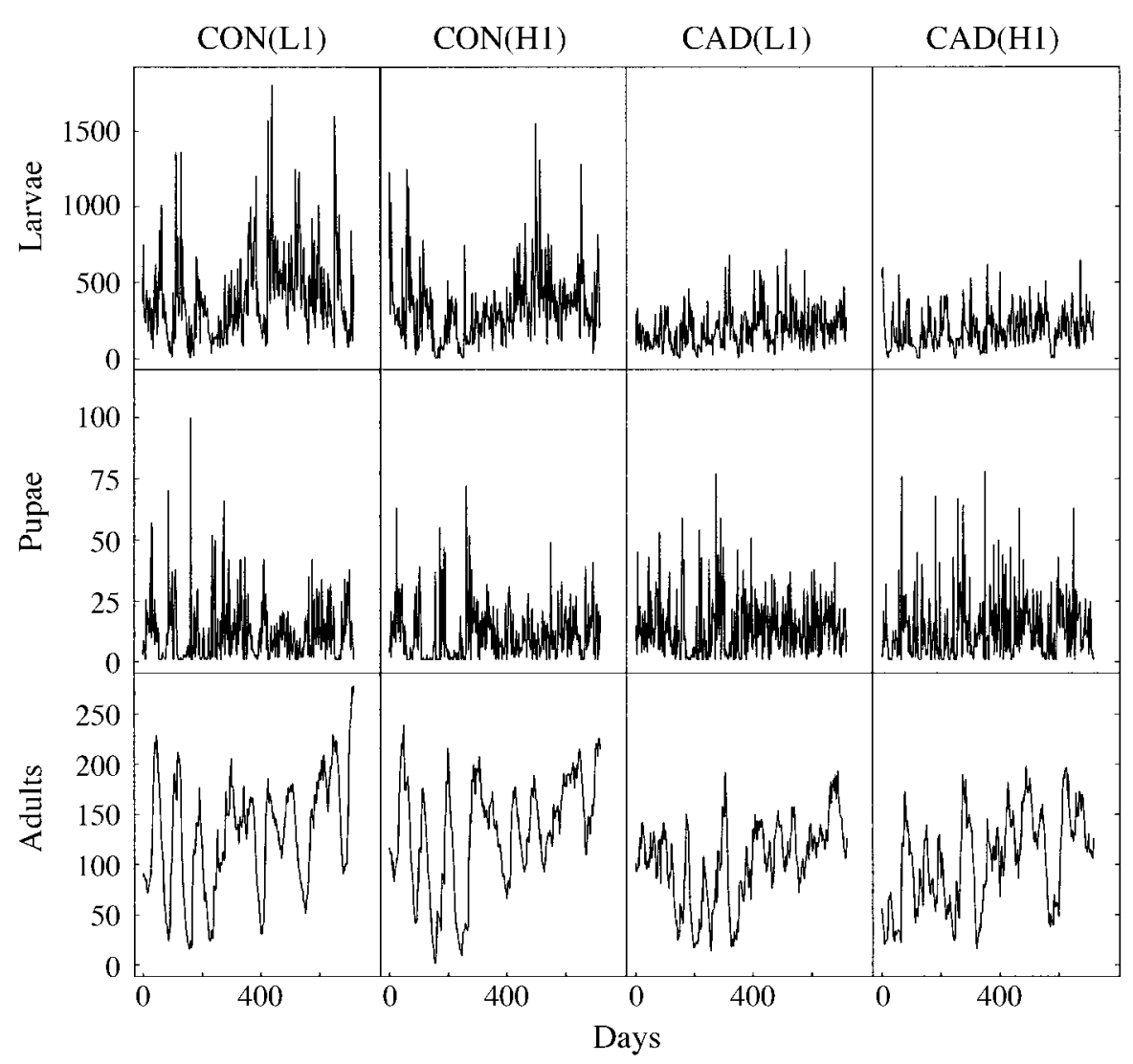

FIG. 2. Stage-specific abundances of four L. sericata populations, counted every two days over a period of $776 \mathrm{~d}$. Abbreviations are: CON, control; CAD, cadmium; L, low density; H, high density; "1" denotes first replicate.

the model, at the expense of making the model more detailed and possibly more complicated. We adopted the latter approach, since it requires only the recorded variables for estimation.

The model to be described consists of a number of equations of the form

$$
N_{t+1, k}=N_{t, k_{0}} \exp \left[\alpha+g_{1}\left(\log N_{t-\tau, k_{1}}\right)+g_{2}\left(\log N_{t-\tau, k_{2}}\right)\right]
$$

where $N_{t, k}$ denotes the $k$ th element of the state vector $\mathbf{N}_{t}$, $\tau \geq 0$ is a time delay, $\alpha$ is a constant, and $g_{1}$ and $g_{2}$ are smooth functions (see the Appendix for a technical definition of smooth functions), one or both of which may be absent. The variables $N_{t, k_{0}}$ and $N_{t+1, k}$ correspond to subsequent age groups in the blowfly life cycle; thus the exponential factor in Eq. 2 represents either rate of survival or rate of reproduction, depending on the context. The per-unit-abundance net growth rate is defined as log $N_{t+1, k}-\log N_{t, k_{0}}$ and is assumed to be an additive function of $\log N_{t-\tau, k_{1}}$ and $\log N_{t-\tau, k_{2}}$ plus noise. Density independence (respectively density dependence) corresponds to having $g_{1} \equiv g_{2} \equiv 0$ (respectively $g_{1} \neq 0$ for at least one function). In the case of density independence, the perunit-abundance rate is simply a constant. In the case of density dependence, the per-unit-abundance rate decreas- es (respectively increases) as a function of $N_{t-\tau, k_{i}}$ when $g_{i}^{\prime}\left(N_{t-\tau, k_{i}}\right)<0$ (respectively $g_{i}^{\prime}\left(\log N_{t-\tau, k_{i}}\right)>$ $0)$, where the prime indicates differentiation.

\section{Stage Structure Representation}

\section{Model assumptions}

For modeling purposes, we make the assumption that the blowfly life history consists of four basic developmental stages: egg/larva, pupa, immature adult and mature adult. Approximate length of each stage is shown in Table 1; some stages have a length that depends on the treatment. Here, we assume that the larval stage lasts for eight days, the pupal stage lasts for 10 $\mathrm{d}$, and the immature adult stage lasts for four days. As a consequence, stage durations are assumed to be density independent. The life span of mature adults has no specified upper limit. A separate egg stage has not been included in the model, in part because data are not available on the number of eggs. Since hatching is completed within roughly one day (Daniels 1994), omitting this stage from the model is not in conflict with a time step of two days. A further modeling assumption is that the demographic rates are identical for all individuals within a given age group.

Different age groups within a life stage are assumed 


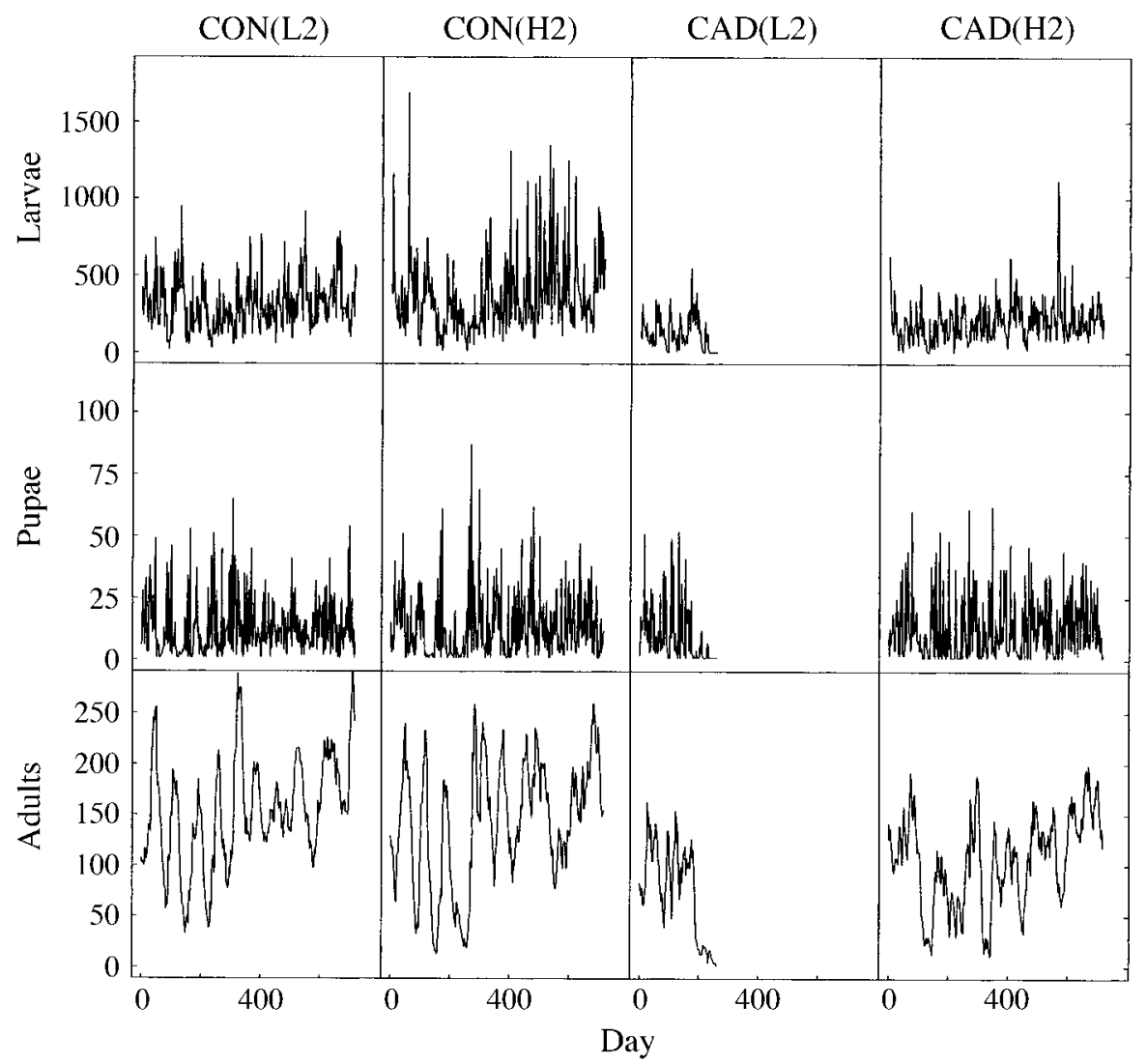

FIG. 3. Stage-specific abundances of four L. sericata populations, counted every two days over a period of $776 \mathrm{~d}$. The population CAD(L2) went extinct after 298 d. See Fig. 2 legend for definitions; "2" denotes second replicate.

to have identical density-dependent structure. The reproductive rate is assumed to depend only on the density of mature adults. Assuming that any effects of density on the larvae manifest themselves on survival in the transition from larva to (viable) pupa, the survival probability of a larva from one age group to the next will be density independent. The proportion of larvae pupating (to viable pupae) at any given time is modeled as a function of the total number of larvae four days before. The latter quantity serves as a proxy (up to a proportionality factor) for the number of larvae in the feeding phase at the time the currently pupating larvae were feeding. This reflects the expectation that density-dependent effects to be found in the larval stage are related to the feeding conditions of the larvae.

Note that, since pupal counts include only the viable pupae, the only way to leave the pupal stage is to become an adult. Since the immature stage accounts for only a small part of the whole adult stage $(\sim 5$ out of $17 \mathrm{~d}$ ), we make the assumption that no deaths occur in the immature adult stage (this greatly simplifies the estimation process). As long as we do not ask for the death rate amongst immature and mature adults separately, but only the overall death rate within the adult stage, little harm is done by making this assumption.

\section{The model}

A stage-structured model for the population dynamics will now be presented. Let

$$
\begin{aligned}
L_{t}^{i} \equiv & \text { population of larvae } d \in[2 i-2,2 i] \text { days old } \\
& \text { at time } t \\
P_{t}^{i} \equiv & \text { population of pupae } d \in[2 i-2,2 i] \text { days old } \\
& \text { at time } t \\
A_{t}^{i} \equiv & \text { population of adults } d \in[2 i-2,2 i] \text { days old } \\
& \text { at time } t
\end{aligned}
$$

where the range of the superscript $i \in\{1, \ldots, 4\}$ in the first definition, $\{1, \ldots, 5\}$ in the second, and $\{1,2,3, \ldots\}$ in the third. Superscripts indicate age groups within a stage (i.e., members differing at most two days in age within a stage). Abundances of immature adults and mature adults at time $t$ are approximated by

$$
\begin{aligned}
A_{t}^{\mathrm{I}} & \left.=\sum_{j=1}^{2} A_{t}^{j} \quad \text { (immature adults at time } t\right) \\
A_{t}^{\mathrm{M}} & =\sum_{j=3}^{\infty} A_{t}^{j} \quad(\text { mature adults at time } t) .
\end{aligned}
$$




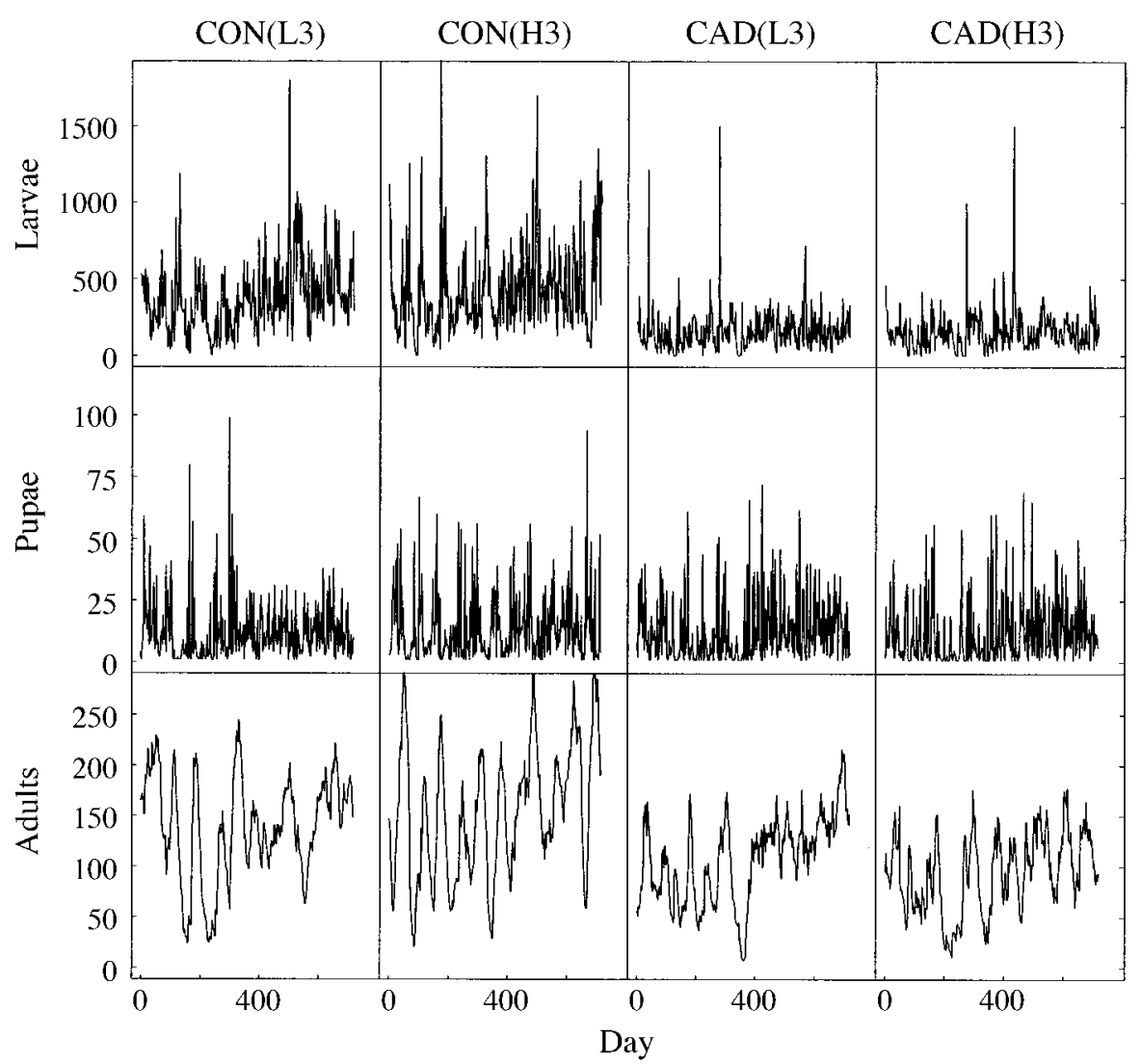

FIG. 4. Stage-specific abundances of four L. sericata populations, counted every two days over a period of 776 d. See Fig. 2 legend for definitions; "3" denotes third replicate.

Furthermore, note that the total size of the larval population at time $t$ is

$$
L_{t}=\sum_{j=1}^{4} L_{t}^{j}
$$

The stage-structured model is given by the following set of equations:

$$
\begin{aligned}
L_{t+1}^{1} & =A_{t}^{\mathrm{M}} \exp \left[\alpha_{\mathrm{L} 1}+f_{\mathrm{L}}\left(\log A_{t}^{\mathrm{M}}\right)\right] \\
L_{t+1}^{i+1} & =L_{t}^{i} \exp \left(\alpha_{\mathrm{L} 2}\right) \quad 1 \leq i \leq 3 \\
P_{t+1}^{1} & =L_{t}^{4} \exp \left[\alpha_{\mathrm{P}}+f_{\mathrm{P}}\left(\log L_{t-1}\right)\right] \\
P_{t+1}^{i+1} & =P_{t}^{i} \quad 1 \leq i \leq 4 \\
A_{t+1}^{2} & =A_{t}^{1}=P_{t-1}^{5} \\
A_{t+1}^{i+1} & =A_{t}^{i} \exp \left[\alpha_{\mathrm{A}}+f_{\mathrm{A} 1}\left(\log A_{t}^{\mathrm{I}}\right)+f_{\mathrm{A} 2}\left(\log A_{t}^{\mathrm{M}}\right)\right] \\
& \quad 2 \leq i<\infty
\end{aligned}
$$

where the smooth functions $\left(f_{\mathrm{L}}, f_{\mathrm{P}}, f_{\mathrm{A} 1}\right.$, and $\left.f_{\mathrm{A} 2}\right)$ and the parameters $\left(\alpha_{\mathrm{L} 1}, \alpha_{\mathrm{L} 2}, \alpha_{\mathrm{P}}\right.$, and $\left.\alpha_{\mathrm{A}}\right)$ correspond to the density-dependent and density-independent components of the demographic rates, respectively. When no confusion can arise, we refer to the parameter values simply as reproduction rate, larval survival rate, and so on.
The particular form of the model in Eqs. 5-10 is similar to, e.g., the larva-pupa-adult (LPA) model (Dennis et al. 1995). However, the latter model is based on only three stages and assumes linear functions inside the exponentials rather than general smooth functions.

\section{Statistical methods}

To determine the demographic rates in Eqs. 5-10, a new set of equations is derived, with the purpose of linking the unknown rates to observed quantities. A combination of nonparametric regression (GAM) and nonlinear regression is used to estimate the densityindependent components (constant terms) and the den-

TABle 1. Approximate time span (in days) of each stage in the life cycle of $L$. sericata.

\begin{tabular}{lcc}
\hline \hline \multicolumn{1}{c}{ Life stage } & $\begin{array}{c}\text { Control } \\
\text { populations }\end{array}$ & $\begin{array}{c}\text { Cadmium } \\
\text { populations }\end{array}$ \\
\hline Egg and larva & 8 & 9 \\
Pupa & $6-12$ & $6-12$ \\
Immature adult & 5 & 5 \\
Mature adult & 12 & 9
\end{tabular}

Note: Some of the numbers in the table depend on larval density, as well as on other factors. 

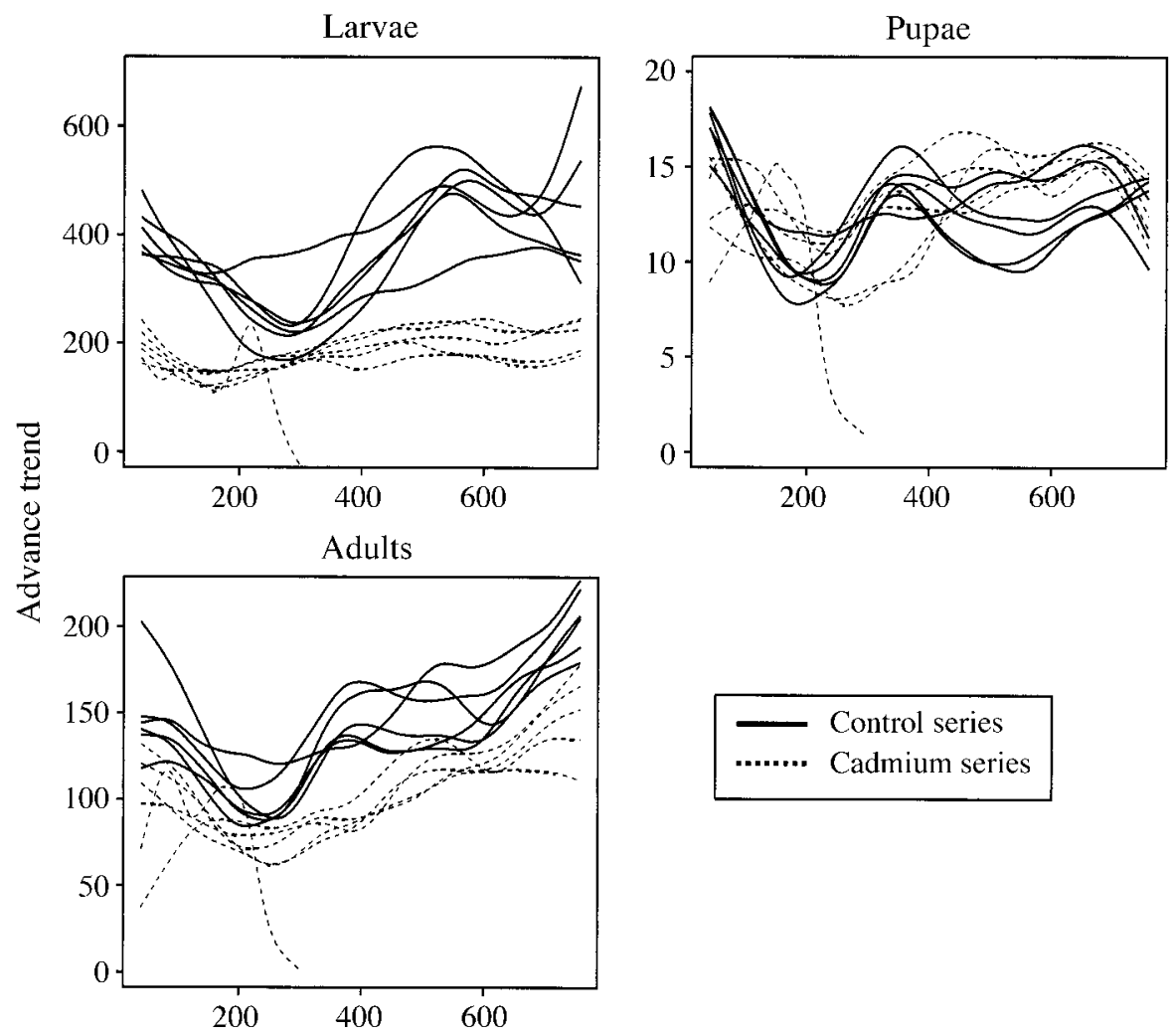

FIG. 5. Nonparametric trend estimates for all the time series, considered as functions of the observation times. Each curve was found by smoothing one of the time series with a cubic smoothing spline using seven degrees of freedom.

sity-dependent components (functions); see the Appendix for more details about the estimation procedure.

To compare parameter values, simple parametric tests such as the $t$ test are not applicable, due to the unknown distributional properties of the parameter estimates. Instead, we resort to using two nonparametric tests, one for testing whether two samples could have come from populations with the same mean (the MannWhitney [MW] $U$ test), and one for testing whether two samples could have come from identical distributions (the Kolmogorov-Smirnov [KS] test). Both tests assume that the samples are mutually independent random samples; since samples correspond to independent populations (trials), this is a reasonable assumption. Formal statistical comparison of function shapes is a difficult problem and will not be treated here (but see Bowman and Azzalini [1997] for a discussion of this topic for models involving a single smooth function). Rather, we emphasize the exploratory nature of our analysis and simply compare function shapes visually.

All the time series analyzed as part of this study have long-term trends (Fig. 5), and most show reduced variability in pupal and adult counts towards the end of the experimental period (Fig. 6). The long-term trends consist of an initial decline phase lasting for $\sim 200$ $300 \mathrm{~d}$ (depending on which life stage we consider), followed by an increase phase lasting an additional 200-300 d. The trends then appear to level off. To examine the effects of the long-term trends on the estimated rates, each time series was split into two halves of equal length, denoted part I and part II, which were analyzed separately.

The model in Eqs. 5-10 was first estimated for four different sets of data, each being the result of combining data from all three replicates of $\mathrm{CON}(\mathrm{L}), \mathrm{CON}(\mathrm{H})$, $\mathrm{CAD}(\mathrm{L})$, or $\mathrm{CAD}(\mathrm{H})$. As a check of consistency between replicates, we also estimated the model for individual replicates. We found that estimates derived from data on single replicates are very similar. Furthermore, the effect on the estimates of initial population density seems negligible.

\section{RESUlts}

\section{Density-independent components of demographic rates}

Estimated density-independent components of the demographic rates are shown in Fig. 7, and a comparison of parameter values is shown in Table 2. Visual examination of Fig. 7 reveals that estimated rates are quite similar between replicates, and indeed within the control populations and within the cadmium populations. 

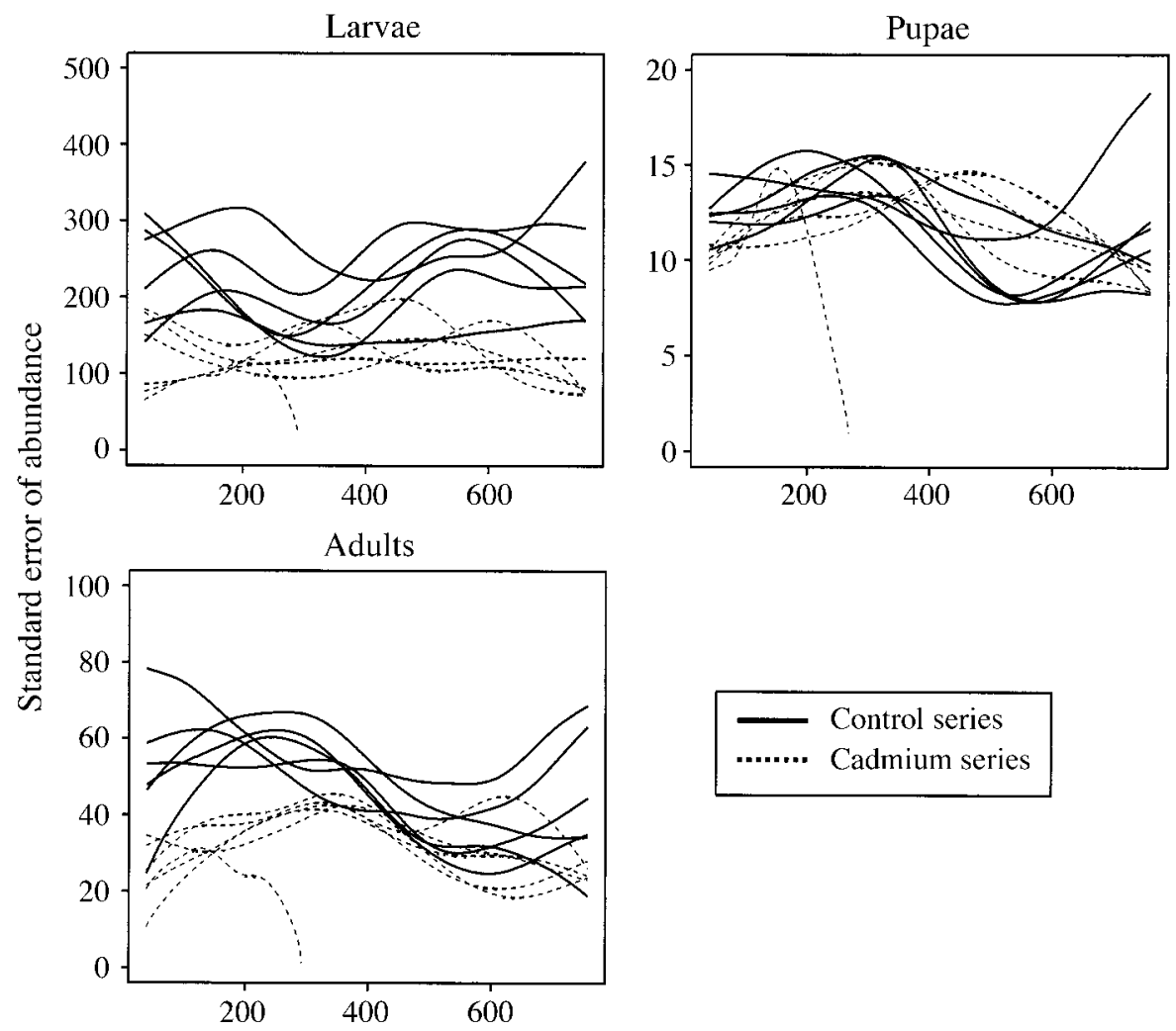

FIG. 6. Nonparametric standard-error estimates for all the time series. Each curve was found by smoothing one of the time series (considered as a function of the observation times), then smoothing the time series of squared residuals from the first smooth, and finally taking the square root. Smooths were computed using a cubic smoothing spline with five degrees of freedom.

Cadmium populations have significantly larger larva-to-adult survival rate $\left(\alpha_{\mathrm{P}}\right)$ and smaller adult survival rate $\left(\alpha_{\mathrm{A}}\right)$ than control populations $\left(p_{\mathrm{MW}}<0.01\right.$ and $p_{\mathrm{KS}}$ $<0.01$ in both cases, both for part I and part II). Cadmium populations have significantly smaller reproductive rate than control populations in part II $\left(p_{\mathrm{MW}}=0.03\right.$ and $\left.p_{\mathrm{KS}}=0.03\right)$, but not in part I.

Reproductive rate $\left(\alpha_{\mathrm{L} 1}\right)$ increases significantly from part I to part II ( $p_{\mathrm{MW}}<0.01$ and $p_{\mathrm{KS}}<0.01$, for both control populations and cadmium populations). For control populations, larva-to-adult survival rate $\left(\alpha_{\mathrm{P}}\right)$ decreases significantly $\left(p_{\mathrm{MW}}<0.01\right.$ and $\left.p_{\mathrm{Ks}}=0.03\right)$ and adult survival rate $\left(\alpha_{\mathrm{A}}\right)$ increases significantly ( $p_{\mathrm{MW}}$ $<0.01$ and $\left.p_{\mathrm{KS}}<0.01\right)$ from part I to part II of the experiment. A change of parameters from part I to part II is consistent with the presence of long-term trends.

\section{Density-dependent components of demographic rates}

Fig. 8 shows function estimates based on all replicates within each of the four experimental groups. Functions are quite similar, even when comparing cadmium populations and control populations. The larvato-adult survival curve $\left(f_{\mathrm{P}}\right)$ is markedly nonlinear, illustrating the usefulness of empirical modeling in discovering the nature of functional relationships. The curve $f_{\mathrm{P}}$ is increasing for low larval densities and (except for part I of CAD $[\mathrm{H}]$ ) decreasing for higher densities. On the other hand, there is no apparent effect of adult density (neither immature nor mature) on the adult survival rate $\left(f_{\mathrm{A} 1}\right.$ and $\left.f_{A 2}\right)$. The estimated reproduction curve $\left(f_{\mathrm{L}}\right)$ decreases markedly with adult density.

Fig. 9 shows plots of function estimates for each of the eleven populations that persisted. There is again a high degree of consistency between the results obtained for different replicates.

\section{DISCUSSION}

The Lucilia sericata data analyzed in this study include both an experimental contrast (control vs. cadmium) and replication, thereby providing an unique opportunity to evaluate methodology that might be useful in analyzing ecological time series. Our analyses demonstrate strong consistency between the estimation results obtained for different replicates of the same experimental treatment. The strong consistency between estimates obtained for different replicates not only emphasizes the effectiveness of the generalized additive model (GAM) methodology, but also high- 


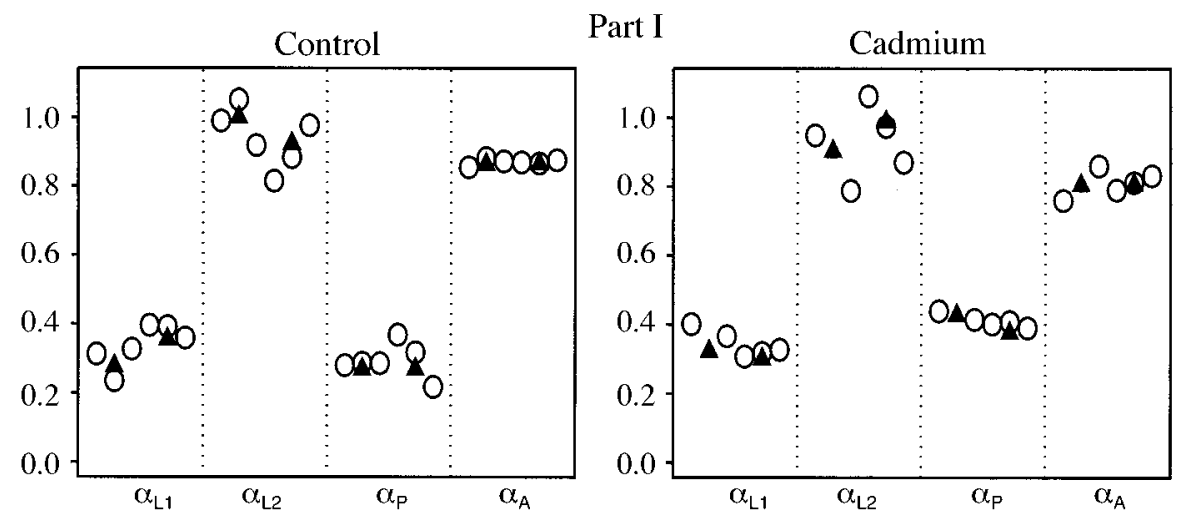

Part II
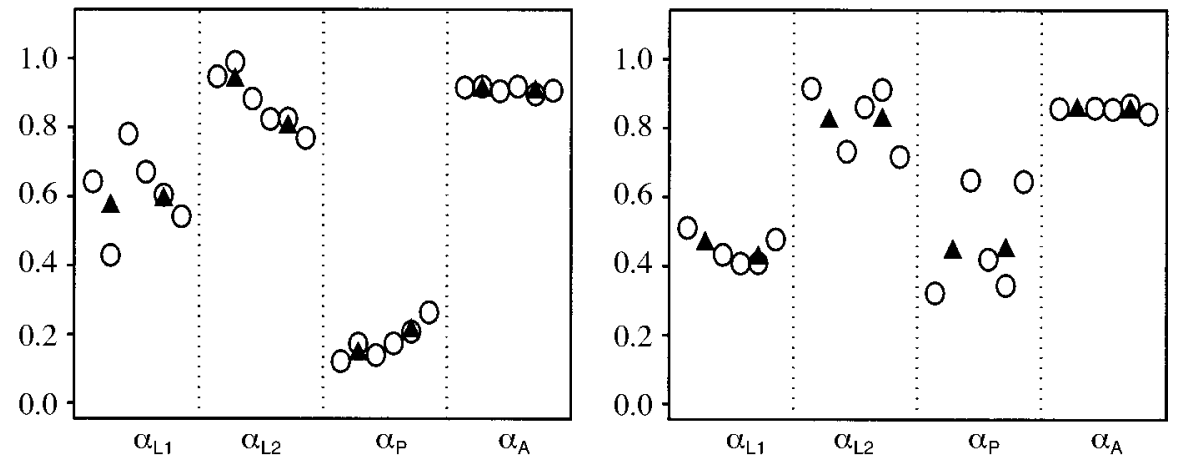

FIG. 7. Estimate of the density-independent component of the demographic rates, for individual populations (shown as circles) and for the four experimental groups (shown as filled triangles), for the model in Eqs. 5-10. For each parameter in each panel, the left-most three (two in the case of the cadmium treatment) circles correspond to the L (low initial density) replicates, and the right-most three circles correspond to the $\mathrm{H}$ (high initial density) replicates. The two filled triangles are pooled estimates for the entire $\mathrm{L}$ group and the entire $\mathrm{H}$ group, respectively. Note that parameter values are plotted as $\exp (\alpha)$ for the various replicates.

lights the obvious value of replication (Smith et al. 2000) in ecological time series studies.

\section{General features of the model}

There is no significant effect of adult density on the adult survival rate $\left(f_{\mathrm{A} 1}\right.$ and $\left.f_{\mathrm{A} 2}\right)$. This result is not surprising, considering that adults are fed ad libitum, and consequently adults supposedly have no need to compete for food. Nevertheless, the estimated reproduction curve $\left(f_{\mathrm{L}}\right)$ decreases markedly with adult density. Pos- sible explanations for this include reduced fecundity due to other kinds of stress experienced by adults at high densities, local mate competition, and the fact that the total reproduction is limited by the area suitable for oviposition (the fresh diet). The negative slope of $f_{\mathrm{L}}$ could also be a delayed effect of competition at the larval stage, which may reduce body size and therefore fecundity of these individuals as adults (Ullyett 1950, Nicholson 1954a). Such an effect could easily have been incorporated into the model, but without appro-

TABLE 2. Tests for equal location of parameters in control and cadmium populations (testing part I and part II separately) and in part I and part II (testing control and cadmium populations separately), using a two-sided alternative; entries are probability values.

\begin{tabular}{|c|c|c|c|c|c|c|c|c|}
\hline \multirow[b]{3}{*}{ Parameter } & \multicolumn{4}{|c|}{ Control vs. cadmium } & \multicolumn{4}{|c|}{ Part I vs. part II } \\
\hline & \multicolumn{2}{|c|}{ Part I } & \multicolumn{2}{|c|}{ Part II } & \multicolumn{2}{|c|}{$\mathrm{CON}$} & \multicolumn{2}{|c|}{ CAD } \\
\hline & $p_{\mathrm{MW}}$ & $p_{\mathrm{KS}}$ & $p_{\mathrm{MW}}$ & $p_{\mathrm{KS}}$ & $p_{\mathrm{MW}}$ & $p_{\mathrm{KS}}$ & $p_{\mathrm{MW}}$ & $p_{\mathrm{KS}}$ \\
\hline$\alpha_{\mathrm{L} 1}$ & 1.00 & 0.97 & 0.03 & 0.03 & $<0.01$ & $<0.01$ & $<0.01$ & $<0.01$ \\
\hline$\alpha_{\mathrm{L} 2}$ & 0.79 & 0.90 & 0.54 & 0.69 & 0.24 & 0.47 & 0.15 & 0.36 \\
\hline$\alpha_{P}$ & $<0.01$ & $<0.01$ & $<0.01$ & $<0.01$ & $<0.01$ & 0.03 & 0.84 & 0.87 \\
\hline$\alpha_{\mathrm{A}}$ & $<0.01$ & 0.03 & $<0.01$ & $<0.01$ & $<0.01$ & $<0.01$ & 0.10 & 0.08 \\
\hline
\end{tabular}

Notes: Probability values are given both for a two-sample Mann-Whitney $U$ test $\left(p_{\mathrm{MW}}\right)$ and for a two-sample KolmogorovSmirnov test $\left(p_{\mathrm{KS}}\right)$. Abbreviations are: CON, control; CAD, cadmium. 

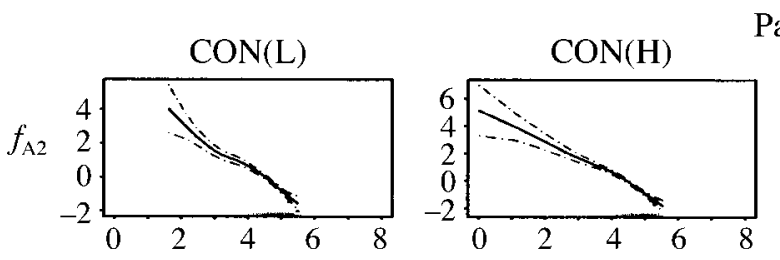

Part I
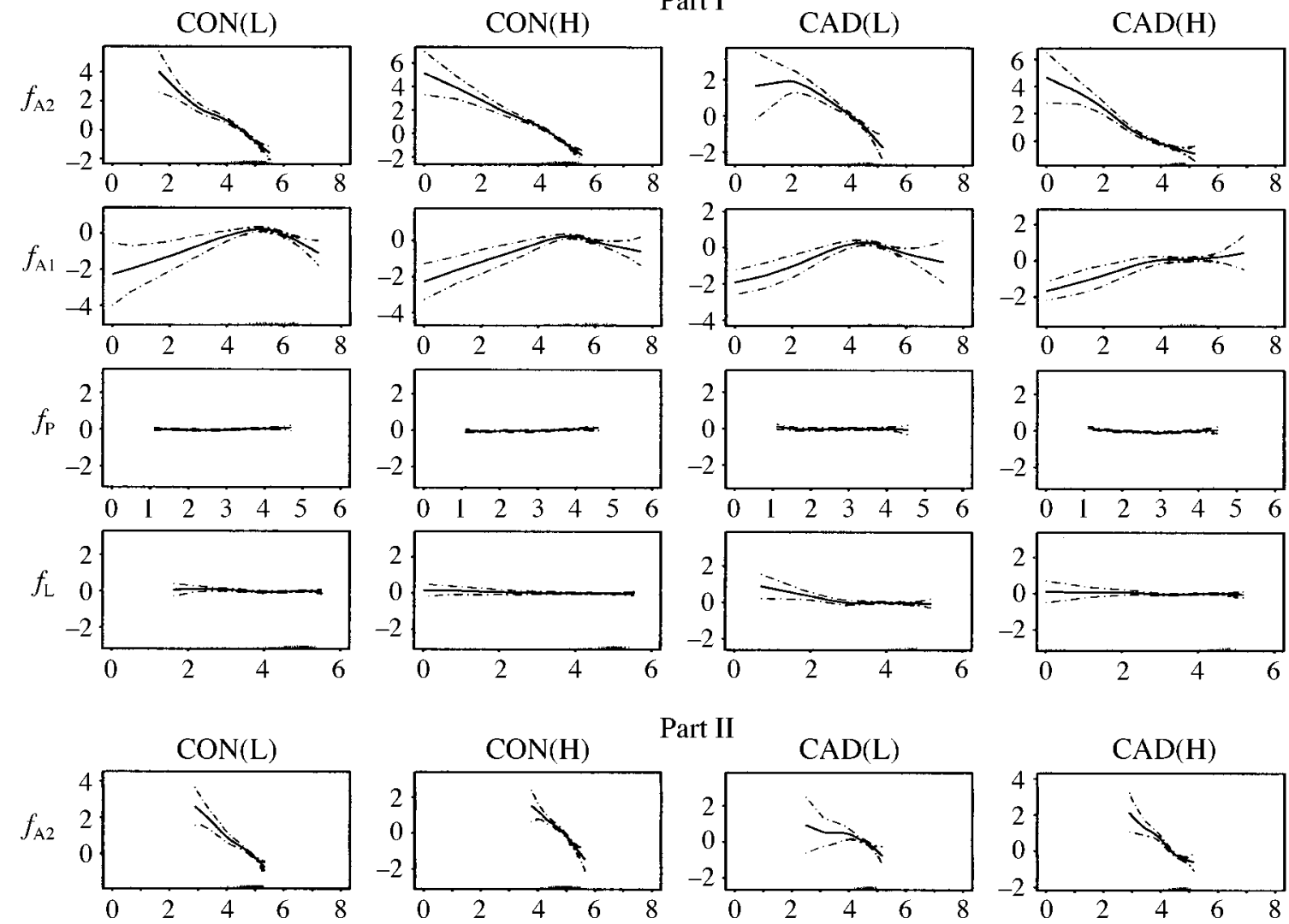

Part II
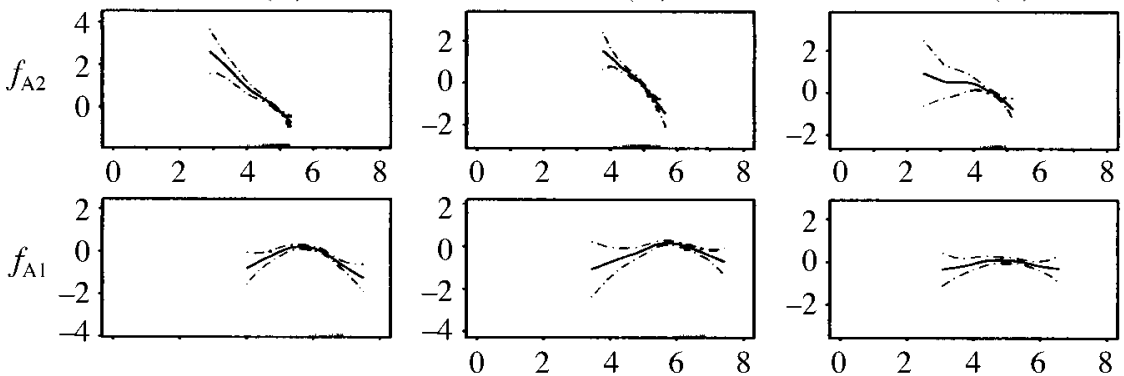

\section{$\mathrm{CAD}(\mathrm{H})$}
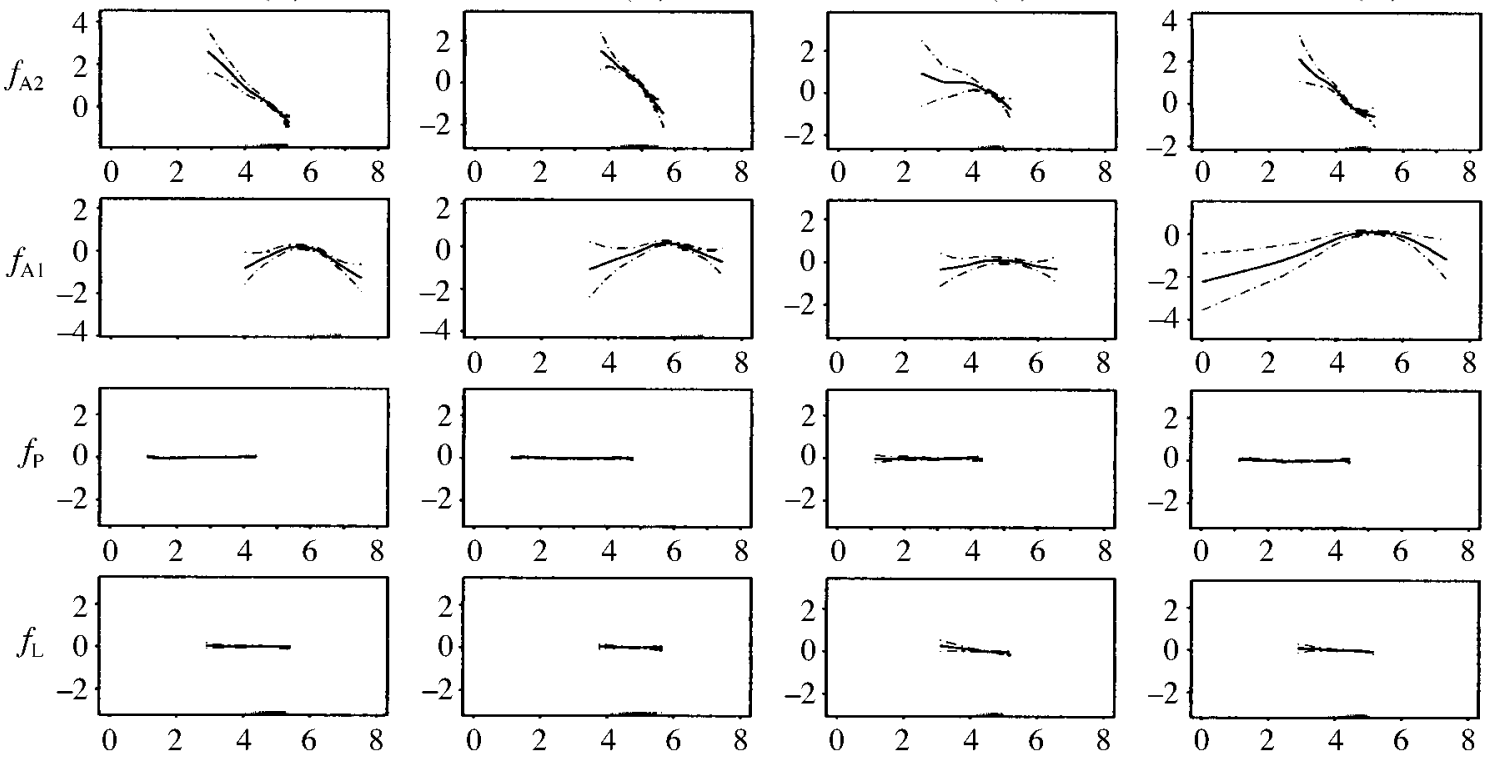

FIG. 8. Estimate of the density-dependent component of the demographic rates, for each of the four experimental groups, for the model in Eqs. 5-10. Approximate 95\% confidence bands are also shown (dotted lines). Argument values are shown as rug plots along the abscissas. Functions are centered (see the Appendix) and are plotted on the same range. Estimates for $\mathrm{CAD}(\mathrm{L})$ are based on data for $\mathrm{CAD}(\mathrm{L} 1)$ and $\mathrm{CAD}(\mathrm{L} 3)$ only.

priate data (requiring additional experiments) we cannot determine whether or not this effect is the real cause for the decrease in the estimated reproduction rate.

Larval competition is known to be an important component of blowfly population dynamics (Nicholson 1950). Based on the observation that reduction in the amount of food per larva leads to reduced growth and survival of L. sericata, Simkiss et al. (1993) concluded that increasing larval densities have negative effects on larval survival. However, we found that increased larval density has a positive effect on larva-to-adult survival $\left(f_{\mathrm{P}}\right)$ at relatively low densities (an Allee effect). This phenomenon, known as facilitation, is common among dipteran larvae and has been described in earlier experiments with blowflies (Ullyett 1950, Wu 1978). At low densities, aggregation serves to increase local temperature and humidity, and thereby the efficiency of external digestion (Ullyett 1950, Hanski 

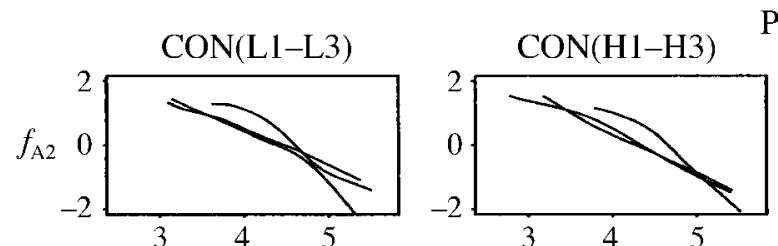

Part I
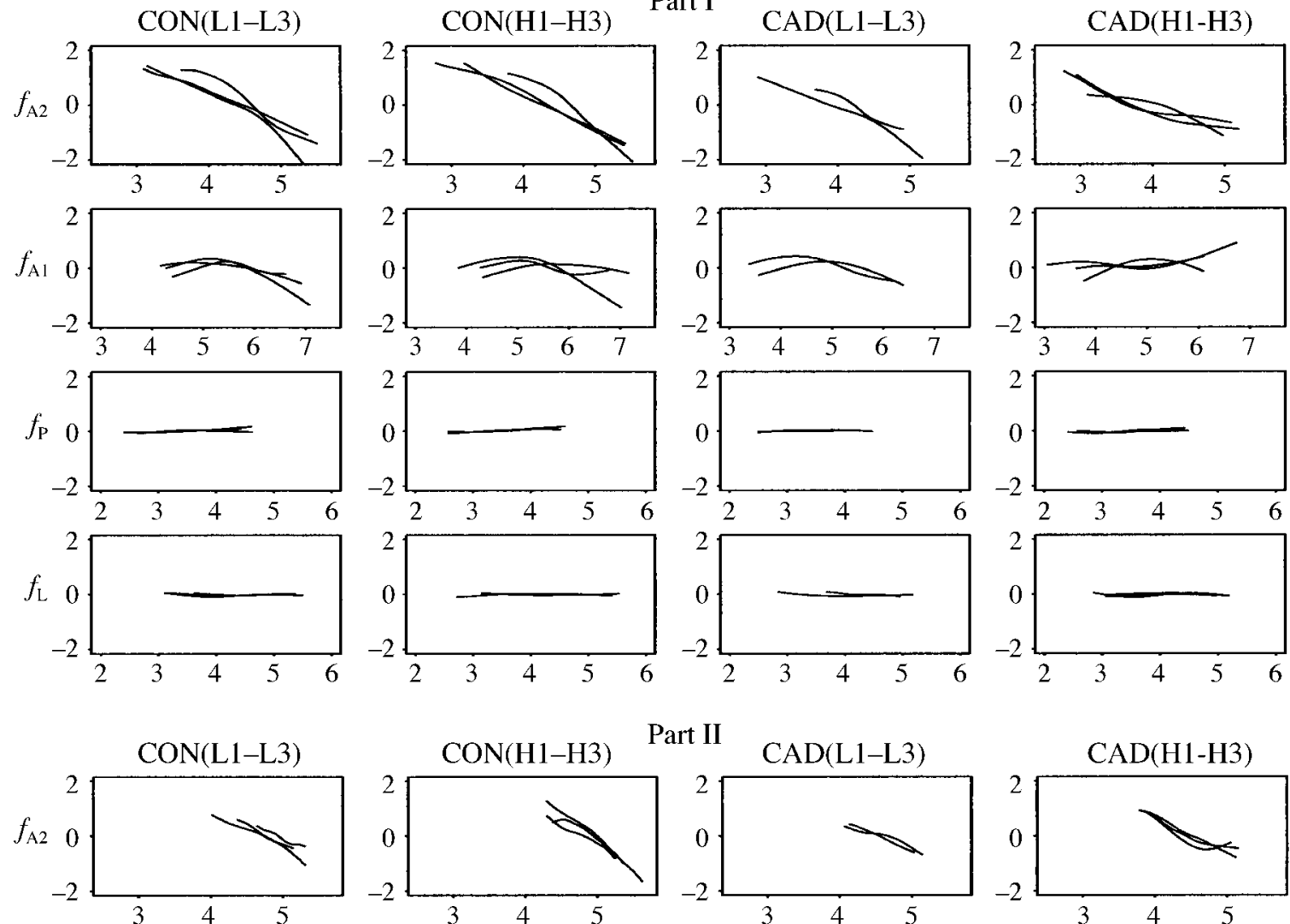

Part II

CAD $(\mathrm{L} 1-\mathrm{L} 3)$
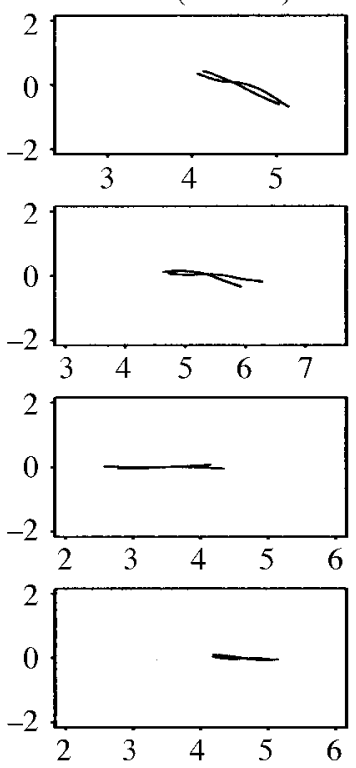

$\mathrm{CAD}(\mathrm{H} 1-\mathrm{H} 3)$
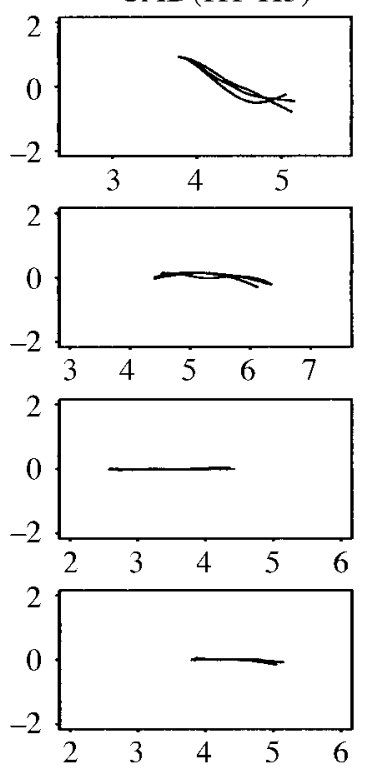

FIG. 9. Estimate of the density-dependent component of the demographic rates, for all 12 populations, for the model in Eqs. 5-10. Functions are centered (see the Appendix) and are plotted on the same range. Estimates for CAD(L2) are not shown.

1987), and may therefore contribute to increased growth and survival of the larvae. Yet, at higher densities, competition results.

\section{Effects of cadmium}

Although cadmium has a significant effect on some demographic rates (larva-to-adult survival rate $\left[\alpha_{\mathrm{P}}\right]$ and adult survival rate $\left[\alpha_{A}\right]$, and for part II also reproduction rate $\left.\left[\alpha_{\mathrm{L} 1}\right]\right)$, the effect on the density-dependent components appears (by visual examination) to be rath- er small. Examining Fig. 8, the estimates of $f_{\mathrm{P}}$ have a peak that is shifted to the left by $\sim 20 \%$ when cadmium is present, indicating an increased effect of larval competition at high larval densities in cadmium populations. Overall, there is lower reproduction and lower survival in the cadmium populations, but also higher larva-to-adult survival. Lower fecundity in cadmium populations may reduce population growth such that larval density does not exceed carrying capacity as much as in controls. The higher larva-to-adult survival 
may thus be a result of reduced density-dependent larval mortality.

\section{Concluding comments}

Nonparametric regression has allowed us to disentangle the density-dependent effects, as well as the density-independent effects, at different stages under various environmental conditions (for another example, see, e.g., Leirs et al. [1997]). Indeed, the nonparametric approach is a very valuable first step in the development of data-based population models. It is by taking such an open-minded view that we are able to detect new (i.e., not previously appreciated) patterns in the way different stages interact. This novel insight provides a basis for more detailed experimental studies (aiming at verifying or rejecting the patterns suggested on the basis of this kind of time series modeling). Analysis that incorporates greater detail of the emerging dynamics will be further facilitated by having a parameterized model, since we then more easily can study the effect of changing the strength of, e.g., the density-dependent effects.

Further work suggested by these results falls into two categories. On the statistical side, the model could be extended to include interaction terms, the significance of which should then be tested in the treatment and the control group. The statistical significance of the effect of cadmium on the population dynamics should be tested formally, following formal testing of common structures for panels of time series (Tong 1990, K.-S. Chan, H. Tong, and N. C. Stenseth, unpublished manuscript). The current results can then be used to develop simpler, parametric models that are easier to analyze mathematically (cf. Smith et al. 2000).

On the experimental side, short-term experiments are needed in order to test hypotheses about model structure. In particular, one may investigate the modeling assumption that different age groups within a life stage have identical density-dependent structure. Forrest (1996) investigated the density dependence from larvae to pupae using even-aged cohorts. However, competition between different larval instars is likely to be asymmetric, and future experiments should examine the effects of both larval age structure and density on adult emergence.

Finally, we argue that this sort of experimental and analytical approach can yield new insights into areas of applied ecology such as ecotoxicology. We noted above that flies in cadmium populations overall have lower fecundity than control flies, hence the cadmium populations do not rise to such high densities and are not as strongly affected by nonlinearities as are the control populations. One consequence of this is that the mean biomass production at the pupal stage is higher for the cadmium than for the control populations (Daniels 1994), even though conven- tional ecotoxicology dogma would predict the opposite: the simple prediction would be that individuals exposed to a toxin such as cadmium should have less energy available for growth and reproduction (i.e., reduced "scope for growth"), hence contributing to a lower average biomass per population. In practice, the interaction of the toxin with the strongly nonlinear density dependence produced an emergent property at the population level that is at variance with the simple scope-for-growth prediction. This highlights the importance of dissecting out the structure of population processes using experimentation and nonparametric regression techniques in order to be able to understand and to predict effects at the population level.

\section{ACKNOWLEDGMENTS}

The analysis reported in this paper has mostly been supported by the Norwegian Science Council (NFR): O. C. Lingjærde was supported by a grant to N. C. Stenseth from NFR (the Ecotoxicology Programme). A. B. Kristoffersen was supported by a grant to N. C. Stenseth from NFR (Strategic University Programme). S. J. Moe was supported by a doctoral stipend from NFR. S. Daniels was supported by three successive small grants from the UK Natural Environment Research Council (NERC) to R. H. Smith and K. Simkiss during the period of data collection. J. M. Read is supported by a grant from the NERC to R. H. Smith. We thank Mike Begon, Steve Ellner, and Ottar Bjørnstad for valuable comments to earlier versions of this paper. We also thank two anonymous reviewers and the associate editor Edward F. Connor for valuable input during the preparation of the final manuscript.

\section{Literature Cited}

Andrewartha, H. G., and L. C. Birch. 1954. The distribution and abundance of animals. University of Chicago Press, Chicago, Illinois, USA.

Begon, M., J. L. Harper, and C. R. Townsend. 1996. Ecology: individuals, populations and communities. Third edition. Blackwell Scientific, Oxford, UK.

Begon, M., S. M. Sait, and D. J. Thompson. 1995. Persistence of a parasitoid-host system-refuges and generation cycles. Proceedings of the Royal Society of London Series B 260:131-137.

Bjørnstad, O. N., M. Begon, N. C. Stenseth, W. Falck, S. M. Sait, and D. J. Thompson. 1998. Population dynamics of the Indian meal moth: demographic stochasticity and delayed regulatory mechanisms. Journal of Animal Ecology 67:110-126.

Bowman, A. W., and A. Azzalini. 1997. Applied smoothing techniques for data analysis. The kernel approach with SPlus illustrations. Clarendon, Oxford, UK.

Caswell, H. 1989a. Matrix population models: constructions, analysis and interpretation. Sinauer, Sunderland, Massachusetts, USA.

Caswell, H. 1989b. Analysis of life table response experiments. 1. Decomposition of effects on population growth rate. Ecological Modelling 46:221-238.

Daniels, S. 1994. Effects of cadmium toxicity on population dynamics of the blowfly Lucilia sericata. Dissertation. University of Reading, UK.

Dennis, B., R. A. Desharnais, J. M. Cushing, and R. F. Constantino. 1995. Nonlinear demographic dynamics: mathematical models, statistical methods, and biological experiments. Ecological Monographs 65:261-281.

Ebenman, B., and B. Pearsson. 1988. Size structured popu- 
lations: ecology and evolution. Springer-Verlag, Berlin, Germany.

Ellner, S. P., and P. Turchin. 1995. Chaos in a noisy world: new methods and evidence from time series analysis. American Naturalist 145:343-374.

Forrest, 1996. Toxins and blowfly populations. Dissertation. University of Leicester, UK.

Gurney, W. S. C., S. P. Blythe, and R. M. Nisbet. 1980. Nicholson's blowflies revisited. Nature 287:17-21.

Gurney, W. S. C., S. P. Blythe, and T. K. Stokes. 1999. Delays, demography and cycles: a forensic study. Advances in ecological research 28:127-144.

Gurney, W. S. C., and R. M. Nisbet. 1998. Ecological dynamics. Oxford University Press, New York, New York, USA.

Gurney, W. S. C., R. M. Nisbet, and J. H. Lawton. 1983. The systematic formulation of tractable single species population models incorporating age-structure. Journal of Animal Ecology 52:479-495.

Hanski, I. 1987. Nutritional ecology of dung- and carrionfeeding insects. Pages 837-884 in: F. Slansky and J. G. Rodriguez, editors. Nutritional ecology of insects, mites, spiders, and related invertebrates. John Wiley and Sons.

Hart, J. D. 1997. Non-parametric smoothing and lack-of-fit tests. Springer, New York.

Hastie, T., and R. Tibshirani. 1990. Generalized additive models. Chapman and Hall, London.

Leirs, H., N. C. Stenseth, J. D. Nichols, J. E. Hines, R. Verhagen, and W. Verheyen. 1997. Stochastic seasonality and nonlinear density-dependent factors regulate population size in an African rodent. Nature 389:176-180.

May, R. M. 1973. Stability and complexity in model ecosystems. Princeton University Press, Princeton, New Jersey, USA.

May, R. M. 1976. Models for single populations. Pages 529 in R. M. May, editor. Theoretical ecology. Blackwell Scientific, Oxford, UK.

Nicholson, A. J. 1950. Population oscillations caused by competition for food. Nature 165:476-477.

Nicholson, A. J. 1954a. Compensatory reactions of populations to stress, and their evolutionary significance. Australian Journal of Zoology 2:1-8.

Nicholson, A. J. 1954b. An outline of the dynamics of animal populations. Australian Journal of Zoology 2:9-65.

Nicholson, A. J. 1957. The self-adjustment of populations to change (with discussion). Cold Spring Harbour Symposia on Quantitative Biology 22:153-173.

Orzack, S. H. 1997. Life-history evolution and extinction. Pages 273-302 in S. Tuljapurkar and H. Caswell, editors. Structured-population models in marine, terrestrial and freshwater systems. Chapman and Hall, New York, New York, USA.

Oster, G. 1977. Internal variables in population dynamics. Lectures in Mathematics in the Life Sciences 8:37-68.
Oster, G. 1981. Predicting populations. American Zoologist 21:831-844.

Readshaw, J. L., and W. R. Cuff. 1980. A model of Nicholson's blowfly cycles and its relevance to predation theory. Journal of Animal Ecology 49:1005-1010.

Readshaw, J. L., and A. C. M. van Gerwen. 1983. Agespecific survival, fecundity and fertility of the adult blowfly Lucilia cuprina in relation to crowding, protein food and population cycles. Journal of Animal Ecology 52:879-887.

Simkiss, K., S. Daniels, and R. H. Smith. 1993. Effects of population density and cadmium toxicity on growth and survival of blowflies. Environmental Pollution 81:41-45.

Smith, R. H., S. Daniels, K. Simkiss, E. D. Bell, S. Ellner, and B. Forrest. 2000. Blowflies as a case study in non-linear population dynamics. Pages $137-172$ in J. N. Perry, R. H. Smith, I. P. Woiwod, and D. Morse, editors. Chaos in real data: the analysis of non-linear dynamics in short ecological time series. Kluwer, Dordrecht, The Netherlands.

Stenseth, N. C., K.-S. Chan, E. Framstad, and H. Tong. 1998. Phase- and density-dependent population dynamics in Norwegian lemmings: interaction between deterministic and stochastic processes. Proceedings of the Royal Society B 265: 1957-1968.

Stenseth, N. C., W. Falck, O. N. Bjørnstad, and C. J. Krebs. 1997. Population regulation in snowshoe hare and Canadian lynx: asymmetric food web configurations between hare and lynx. Proceedings from the National Academy of Sciences USA 94:5147-5152.

Sugihara, G., and R. M. May. 1990. Non-linear forecasting as a way of distinguishing chaos from measurement error in time series. Nature 344:734-741.

Takens, F. 1994. Analysis of non-linear time series with noise. Technical Report, 20 March 1994 Department of Mathematics, Groningen University, Groningen, The Netherlands.

Tong, H. 1990. Non-linear time series: a dynamical system approach. Oxford University Press, Oxford, UK.

Tong, H. 1995. A personal overview of non-linear time-series analysis from a chaos perspective, with discussions and comments. Scandinavian Journal of Statistics 22:399-446.

Turchin, P. 1995. Population dynamics. Pages 19-40 in N. Cappuccino and P. Price, editors. Academic Press, New York, New York, USA.

Ullyett, G. C. 1950. Competition for food and allied phenomena in sheep-blowfly populations. Philosophical Transactions of the Royal Society of London B 234:77-174.

Wiesenfeld, K., and F. Moss. 1995. Stochastic resonance and the benefits of noise: from ice ages to crayfish and squids. Nature 373:33-36.

Wu, Y. C. 1978. An experimental and theoretical study of population cycles of the blowfly, Phaenicia sericata (Calliphoridae, in a laboratory ecosystem. Dissertation. University of California-Berkeley, California, USA.

\section{Nonparametric curve estimation}

The model in Eqs. 5-10 involves several unobserved state variables (e.g., $L_{t+1}^{i}, i=1,2,3,4$ ), which makes the individual equations unsuitable for estimation of unknown parameters and functions. Here, we derive a new set of equations involving observed state variables only (i.e., actual counts). Nonparametric additive regression and nonlinear regression can then be used to estimate unknowns. We first prove the following result.

Theorem.-Suppose Eqs. 5-10 hold. Then we have

\section{APPENDIX}

$$
\begin{aligned}
P_{t+1}^{1}= & A_{t-4}^{\mathrm{M}} \exp \left(\alpha+f_{\mathrm{L}}\left(\log A_{t-4}^{\mathrm{M}}\right)+f_{\mathrm{P}}\left(\log L_{t-1}\right)\right) \\
A_{t+1}^{\mathrm{M}}= & \left(A_{t}^{\mathrm{M}}+P_{t-6}^{1}\right) \\
& \times \exp \left(\alpha_{\mathrm{A}}+f_{\mathrm{A} 1}\left(\log A_{t}^{\mathrm{I}}\right)+f_{\mathrm{A} 2}\left(\log A_{t}^{\mathrm{M}}\right)\right) \\
L_{t+1}= & \exp \left(-\alpha_{\mathrm{P}}\right) \sum_{s=0}^{3} P_{t+s+2}^{1} \\
& \times \exp \left(-s \alpha_{\mathrm{L} 2}-f_{\mathrm{P}}\left(\log L_{t+s}\right)\right)
\end{aligned}
$$




$$
\alpha=\alpha_{\mathrm{L} 1}+3 \alpha_{\mathrm{L} 2}+\alpha_{\mathrm{P}} .
$$

Proof.-1) Eq. A.1 follows by applying Eq. 6 repeatedly to the right hand side of Eq. 7, and then applying Eq. 5 to the result.

2) Eq. A.2 holds because

$$
\begin{aligned}
A_{t+1}^{\mathrm{M}} & =\sum_{i \geq 2} A_{t+1}^{i+1} \\
& =\sum_{i \geq 2} A_{t}^{i} \exp \left[\alpha_{\mathrm{A}}+f_{\mathrm{A} 1}\left(\log A_{t}^{\mathrm{I}}\right)+f_{\mathrm{A} 2}\left(\log A_{t}^{\mathrm{M}}\right)\right] \\
& =\left(A_{t}^{\mathrm{M}}+A_{t}^{2}\right) \exp \left[\alpha_{\mathrm{A}}+f_{\mathrm{A} 1}\left(\log A_{t}^{\mathrm{I}}\right)+f_{\mathrm{A} 2}\left(\log A_{t}^{\mathrm{M}}\right)\right] \\
& =\left(A_{t}^{\mathrm{M}}+P_{t-6}^{1}\right) \exp \left[\alpha_{\mathrm{A}}+f_{\mathrm{A} 1}\left(\log A_{t}^{\mathrm{I}}\right)+f_{\mathrm{A} 2}\left(\log A_{t}^{\mathrm{M}}\right)\right] .
\end{aligned}
$$

3) To show Eq. A.3, observe first that

$$
L_{t+1}=\sum_{s=0}^{3} L_{t+1}^{4-s}=\sum_{s=0}^{3} L_{t+s+1}^{4} \exp \left(-s \alpha_{\mathrm{L} 2}\right) .
$$

By using Eq. 7 we find that

$$
L_{t+s+1}^{4}=P_{t+s+2}^{1} \exp \left[-\alpha_{\mathrm{P}}-f_{\mathrm{P}}\left(\log L_{t+s}\right)\right]
$$

and by substituting for $L_{t+s+1}^{4}$ in Eq. A.4 using Eq. A.5, we arrive at Eq. A.3.

Eqs. A.1-A.3 refer only to actually observed state variables ( $L_{t}, P_{t}^{1}, A_{t}$, and quantities that can be derived from these). The number of immature adults $A_{t+1}^{\mathrm{I}}$ can be computed from

$$
A_{t+1}^{\mathrm{I}}=A_{t+1}^{1}+A_{t+2}^{2}=A_{t+1}^{1}+A_{t}^{1}=P_{t-4}^{1}+P_{t-5}^{1}
$$

and the number of mature adults can be found from $A_{t+1}^{\mathrm{M}}=$ $A_{t+1}-A_{t+1}$.

Log-transforming Eqs. A.1 and A.2 and imposing an additive noise structure, we have

$$
\begin{aligned}
\log \left(\frac{P_{t+1}^{1}}{A_{t-4}^{\mathrm{M}}}\right)= & \alpha+f_{\mathrm{L}}\left(\log A_{t-4}^{\mathrm{M}}\right) \\
& +f_{\mathrm{P}}\left(\log L_{t-1}\right)+\varepsilon_{t}^{(1)} \\
\log \left(\frac{A_{t+1}^{\mathrm{M}}}{A_{t}^{\mathrm{M}}+P_{t-6}^{1}}\right)= & \alpha_{\mathrm{A}}+f_{\mathrm{A} 1}\left(\log A_{t}^{\mathrm{I}}\right) \\
& +f_{\mathrm{A} 2}\left(\log A_{t}^{\mathrm{M}}\right)+\varepsilon_{t}^{(2)}
\end{aligned}
$$

where $\varepsilon_{t}^{(i)}, t=1,2, \ldots$, is a white noise process, i.e., a sequence of zero-mean uncorrelated random variables with a common variance $\sigma_{i}^{2}$, for $i=1,2$. By imposing noise on the model, we acknowledge that the original Eqs. 5-10 should only be considered approximate relationships, due to environmental and demographic stochastic forces.

Eqs. A.6 and A.7 are additive models (see, e.g., Hastie and Tibshirani [1990]) for which unknowns can be estimated by minimizing a penalized least-squares problem. For example, in Eq. A. 6 the estimates for $\alpha, f_{\mathrm{L}}$, and $f_{\mathrm{P}}$ can be found by minimizing

$$
\begin{aligned}
& \sum_{t}\left\{\log \left(\frac{P_{t+1}^{1}}{A_{t-4}^{\mathrm{M}}}\right)-\alpha-f_{\mathrm{L}}\left(\log A_{t-4}^{\mathrm{M}}\right)-f_{\mathrm{P}}\left(\log L_{t-1}\right)\right\}^{2} \\
& +\lambda_{1} \int_{-\infty}^{\infty}\left\{f_{\mathrm{L}}^{\prime \prime}(t)\right\}^{2} d t+\lambda_{2} \int_{-\infty}^{\infty}\left\{f_{\mathrm{P}}^{\prime \prime}(t)\right\}^{2} d t
\end{aligned}
$$

over $\alpha$ and all functions $f_{\mathrm{L}}$ and $f_{\mathrm{P}}$ such that the integrals exist. The result is an estimate $\hat{\alpha}$ for the level and estimates $\hat{f}_{\mathrm{P}}(x)$ and $\hat{f}_{\mathrm{L}}(x)$ for the density-dependent effects $f_{\mathrm{P}}(x)$ and $f_{\mathrm{L}}(x)$. Technically, function estimates may be shown to be natural cubic smoothing splines (two-times continuously differentiable piece-wise cubic polynomials); see Hastie and Tibshirani (1990) for details.

The nonnegative penalty terms (i.e., the integrals) are zero only for functions with no curvature (i.e., linear functions), and they attain increasingly larger positive values with increasing curvature of the functions. The smoothing parameters $\lambda_{1}>0$ and $\lambda_{2}>0$ determine the trade-off between goodness-of-fit (represented by the sum-of-squares term) and smoothness (represented by the penalty terms). As $\lambda_{i} \rightarrow \infty$, the corresponding function estimate approaches a linear fit. A useful reparameterization of a smoothing parameter $\lambda$ is in terms of the degrees of freedom $\operatorname{df}(\lambda)$. In general, $\operatorname{df}(\lambda)$ is a strictly decreasing function of $\lambda$, and we have $\operatorname{df}(\lambda) \geq 2$ and $\operatorname{df}(\lambda) \rightarrow 2$ as $\lambda \rightarrow \infty$.

S-PLUS was used to estimate Eqs. A.6 and A.7, using five degrees of freedom for each function. Each equation was fitted by a call to the GAM software of the form $\operatorname{gam}(\mathbf{z} \sim 1$ $+s(\mathbf{x}, 5)+s(\mathbf{y}, 5))$, where $\mathbf{z}$ is the vector of responses (the left-hand side of Eq. A.6 or A.7) and $\mathbf{x}, \mathbf{y}$ are the vectors of covariates $\left(\log A_{t-4}^{\mathrm{M}}\right.$ and $\log L_{t-1}$ for Eq. A.6, and similarly for Eq. A.7).

For identifiability, the functions in these equations are assumed to be centered, i.e.,

$$
\begin{aligned}
\sum_{t} f_{\mathrm{L}}\left(\log A_{t-4}^{\mathrm{M}}\right) & =0 & \sum_{t} f_{\mathrm{P}}\left(\log L_{t-1}\right) & =0 \\
\sum_{t} f_{\mathrm{A} 1}\left(\log A_{t}^{\mathrm{I}}\right) & =0 & \sum_{t} f_{\mathrm{A} 2}\left(\log A_{t}^{\mathrm{M}}\right) & =0 .
\end{aligned}
$$

Estimates for $\alpha$ and $\alpha_{\mathrm{A}}$ in Eqs. A.6 and A.7 are simply the sample averages of the corresponding left-hand sides. In order to estimate the remaining parameters $\alpha_{\mathrm{P}}, \alpha_{\mathrm{L} 1}$ and $\alpha_{\mathrm{L} 2}$, we minimized the squared difference between the two sides of Eq. A.3 with respect to $\alpha_{\mathrm{P}}$ and $\alpha_{\mathrm{L} 2}$. This is a nonlinear (deterministic) regression problem, and we used the routine nls() in S-PLUS to obtain the estimates. Finally, $\alpha_{\mathrm{L}}$ is determined from the given estimates and the relation $\alpha_{\mathrm{L} 1}=\alpha-3 \alpha_{\mathrm{L} 2}-$ $\alpha_{\mathrm{P}}$. S-PLUS code is available as Supplementary Material.

\section{SUPPLEMENTARY MATERIAL}

The S-PLUS code used to obtain the estimates presented in the paper is available in ESA's Electronic Data Archive: Ecological Archives: E082-027. 